\title{
Epidemiology, Treatment Patterns, and Outcomes of Metastatic Soft Tissue Sarcoma in a Community-Based Oncology Network
}

\author{
Clara Chen, ${ }^{1}$ Rohit Borker, ${ }^{2}$ James Ewing, ${ }^{1,3,4,5}$ Wan-Yu Tseng, ${ }^{1}$ Michelle D. Hackshaw, \\ Shanmugapriya Saravanan, ${ }^{1}$ Rahul Dhanda, ${ }^{1}$ and Eric Nadler ${ }^{1,3,4,5,6}$ \\ ${ }^{1}$ Department of Information Technology, Health Economics and Outcomes Research, McKesson Specialty Health, \\ The Woodlands, TX, USA \\ ${ }^{2}$ GlaxoSmithKline, Philadelphia, PA 19112, USA \\ ${ }^{3}$ Texas Oncology, Dallas, TX, USA \\ ${ }^{4}$ Baylor Charles A. Sammons Cancer Center, Dallas, TX, USA \\ ${ }^{5}$ Baylor University Medical Center at Dallas, Dallas, TX, USA \\ ${ }^{6}$ Texas Oncology, Baylor Sammons Cancer Center, 3410 Worth Street, Dallas, TX 75246, USA
}

Correspondence should be addressed to Eric Nadler; eric.nadler@usoncology.com

Received 22 August 2013; Revised 13 December 2013; Accepted 15 December 2013; Published 12 January 2014

Academic Editor: Charles Catton

Copyright (C) 2014 Clara Chen et al. This is an open access article distributed under the Creative Commons Attribution License, which permits unrestricted use, distribution, and reproduction in any medium, provided the original work is properly cited.

\begin{abstract}
Purpose. To assess epidemiology, treatment patterns, and outcomes of metastatic soft tissue sarcoma (mSTS) patients in USA community oncology practices. Methods. This retrospective, descriptive study used US Oncology's iKnowMed electronic health records database. Adults ( $\geq 18$ years) with $\mathrm{mSTS}$ and at least two visits between July 2007 and June 2010 were included. Key outcomes were practice patterns, overall survival (OS), and progression-free survival (PFS). Results. $363 \mathrm{mSTS}$ patients (174 treated and 189 untreated) met the prespecified exclusion/inclusion criteria. The most common subtypes were leiomyosarcoma $(n=104$; $29 \%)$, liposarcoma $(n=40 ; 11 \%)$, and synovial sarcoma $(n=12 ; 3 \%)$; the remainder $(n=207 ; 57 \%)$ comprised 27 histologic subtypes. Treated patients were younger and had lower ECOG scores; $75 \%$ and $25 \%$ received first-line combination or monotherapy, respectively. Median OS of treated and untreated patients was 22 and 17 months, respectively, and 29 months in patients with the three most common subtypes. Before controlling for effects of covariates, younger age and lower ECOG scores were associated with better OS and PFS. Conclusion. This study provides insights into mSTS epidemiology, treatment patterns, and outcomes in a large community-based oncology network. These results warrant further studies with larger cohorts.
\end{abstract}

\section{Introduction}

Soft tissue sarcomas (STS) are rare mesenchymal tumors that account for $1 \%$ of adult cancers [1-7] and comprise over 50 different histologic subtypes that differ in pathogenesis and outcomes $[1-3,6,7]$. Collectively, they are associated with a mortality rate of over 4,000 patients per year [2]. The treatment of STS is dependent upon several factors, including histologic subtype, disease stage, and patient performance status; the treatment options include surgery, radiotherapy, and/or chemotherapy [1, 3, 6-10]. Although localized resected disease can often be cured, the prognosis of patients with metastatic STS (mSTS) remains poor, with median survival of approximately one year $[1,3,6,11-15]$. Good prognostic factors for mSTS include younger patients with good performance status and low tumor grade $[3,6,16]$.

Most of our insights regarding factors that influence the outcomes following chemotherapy in mSTS have been obtained mainly from clinical trials [11-14, 16]. The purpose of this study was to gain an improved understanding of the "realworld" epidemiology as well as treatment patterns and outcomes of mSTS in the setting of community oncology clinics.

\section{Methods}

Data was obtained from the McKesson Specialty Health (MSH)/US Oncology (USON) iKnowMed (iKM) electronic health record (EHR) and electronic chart review. The study 
period was from July 1, 2007, to June 30, 2010, with followup through June 30,2011 . Adults ( $\geq 18$ years) with mSTS were included if they received care at practice sites with the full capabilities of the iKM EHR system and had at least two visits during the study period. Patients were excluded if during the study period they were diagnosed with or treated for a primary cancer other than mSTS or were enrolled in a randomized clinical trial. Electronic chart review was conducted to validate and supplement EHR data for critical parameters, including cell morphology and histology. For the measurement of progression-free survival (PFS), an escalation in the line of therapy (LOT) from first-line to secondline was used as a proxy measurement for disease progression. Thus, PFS in this study was defined as the time in months from the initiation of first-line chemotherapy to the initiation of second-line chemotherapy, or death from any cause, whichever occurred first. Patients who were progression-free were censored at the end of the study follow-up period or at the date they were last known to be alive, whichever occurred first.

Descriptive analyses were conducted on patient demographics and clinical characteristics, as well as treatment patterns. Overall survival (OS) and PFS were estimated using Kaplan-Meier plots. Mean and median survival time and survival rates were derived with $95 \%$ confidence intervals (CI). SAS 9.2 and STATA 11.2 were used for data management and statistical analysis.

\section{Results}

3.1. Study Population. In the iKnowMed EHR, 4,245 individuals had histologically confirmed STS, of which 1,286 (30\%) had metastatic disease. Supplementary Figure 1 depicts the patient consort diagram; based upon the inclusion/exclusion criteria employed, we selected $363 \mathrm{mSTS}$ patients for further evaluation: 174 of the 329 treated and 189 of the 957 untreated patients (see Supplementary Material available online at http://dx.doi.org/10.1155/2014/145764).

Table 1 describes the demographic and clinical characteristics of our study sample. The mean age (standard deviation) at diagnosis of mSTS in the entire study population was 61 (16) years. Forty-eight percent were female and 52\% were male. Based on the histology of their tumors, the study population ( $n=363$ ) was categorized into four major groups: those with leiomyosarcoma ( $n=104 ; 29 \%)$, liposarcoma $(n=40 ; 11 \%)$, and synovial sarcoma $(n=12 ; 3 \%)$, and the remainder were designated as "other" ( $n=207 ; 57 \%)$ as they comprised subjects with 27 histologic subtypes, each with small sample sizes. The frequency of the histology subtypes was similar in treated and untreated patients (Table 1). We adopted this categorization schema as these were the most prevalent groups based on histology, and recent studies used similar groupings [17]. The most common primary tumor sites in the treated population were the extremities $(33 \%)$, retroperitoneal (19\%), and the trunk and viscera (14\%).

Among treated and untreated patients with available clinical data, presentation in both groups was most commonly with stage IV disease ( $54 \%$ and $67 \%$, resp.), grade 3 tumor (69\% each), resectable tumor (50\% and $61 \%$, resp.), and
ECOG score of 1 (49\% and 47\%, resp.) (Table 1). Treated patients were younger (median $=58 \mathrm{yr}$ ) than untreated patients (median $=65 \mathrm{yr}$ ) and the overall ECOG performance at study entry between treated and untreated patients differed (Table 1). In addition, male patients were more likely to receive chemotherapy when compared with female patients (60\% versus $40 \%$, resp.).

3.2. Treatment Patterns. Of the 174 treated patients, 173 (99\%) and 42 (24\%) received first- and second-line therapies, respectively, and only $14(8 \%)$ continued with third-line therapy. (Note: one of the 174 treated patients received second-line therapy as initial therapy.) The most frequently used chemotherapy regimens in first-, second-, and third-line therapies were as follows: for first-line chemotherapy, doxorubicin plus ifosfamide (29\%), docetaxel plus gemcitabine $(24 \%)$, or doxorubicin alone $(12 \%)$ was used. For second-line chemotherapy, docetaxel plus gemcitabine (52\%) was used the most, followed by doxorubicin alone (17\%). For thirdline chemotherapy, liposomal doxorubicin alone $(29 \%)$ or docetaxel plus gemcitabine $(21 \%)$ was given.

Of the treated patients, 64 (37\%) received monotherapy, whereas 135 (78\%) received combination therapy; approximately $14 \%$ received both monotherapy and combination therapies. Among those receiving first-line therapy $(n=173)$, $28 \%$ received monotherapy and $72 \%$ combination therapy, and similar proportions were observed for those receiving second-line therapy (29\% and $71 \%$, resp.). Of the 14 patients who continued chemotherapy into third-line, 57\% and $43 \%$ received monotherapy and combination therapy, respectively. These data suggested that among those receiving first- or second-line chemotherapies, a greater proportion received combination compared with monotherapy. In contrast, the reverse pattern was observed in those receiving third-line therapy.

The following treatment patterns were observed. Among the 49 patients who received monotherapy as first-line therapy, doxorubicin (43\%), gemcitabine (27\%), and liposomal doxorubicin (14\%) were the more frequently used agents, while among the 126 patients receiving first-line combination therapy the most common regimens were doxorubicin plus ifosfamide (41\%), docetaxel plus gemcitabine (35\%), and doxorubicin plus dacarbazine (6\%). Among the 12 patients who received monotherapy as second-line therapy, doxorubicin (58\%) was most often used, while in the 30 subjects receiving combination second-line therapy, docetaxel plus gemcitabine $(73 \%)$ was used most frequently. Ninety-five patients received anthracycline-containing first-line therapy, and of these 37 continued with second-line chemotherapy; among these patients docetaxel plus gemcitabine (70\%) was most commonly used.

We examined the usage of monotherapy versus combination therapy according to histology subtype (Supplementary Table 1). Among those receiving combination $(n=129)$ and monotherapy $(n=50), 56 \%$ and $40 \%$, respectively, were patients with the top three subtypes. Reflecting that a lower proportion of patients with the other subtypes received combination therapy, 72 of the 92 subjects with the top three subtypes $(78.3 \%)$ versus 57 of the 87 individuals with 
TABLE 1: Demographic and clinical characteristics of treated and untreated metastatic STS patients.

\begin{tabular}{|c|c|c|c|c|}
\hline Characteristic & Total $(N=363)$ & Treated patients $(N=174)$ & Untreated patients $(N=189)$ & $P$ \\
\hline \multicolumn{5}{|c|}{ Age at treatment, $N(\%)$} \\
\hline Mean (SD) & $61(16)$ & $58(14)$ & $63(17)$ & 0.0031 \\
\hline Median (range) & $62(18,91)$ & $58(19,90)$ & $65(18,91)$ & 0.0005 \\
\hline$<65$ & $203(60)$ & $109(63)$ & $94(50)$ & \multirow{3}{*}{$<0.0001$} \\
\hline $65-75$ & $76(21)$ & $42(24)$ & $34(18)$ & \\
\hline$\geq 75$ & $84(23)$ & $23(13)$ & $61(32)$ & \\
\hline \multicolumn{5}{|l|}{ Gender, $N(\%)$} \\
\hline Female & $175(48)$ & $70(40)$ & $105(56)$ & \multirow{2}{*}{0.0035} \\
\hline Male & $188(52)$ & $104(60)$ & $84(44)$ & \\
\hline \multicolumn{5}{|l|}{ BMI, $N(\%)$} \\
\hline Mean (SD) & $28.1(6.3)$ & $28.5(5.9)$ & $27.8(6.8)$ & 0.2742 \\
\hline Median (range) & $26.8(15.4,52.1)$ & $27.5(17.1,49.5)$ & $26.4(15.4,52.1)$ & 0.0624 \\
\hline Underweight & $6(2)$ & $3(2)$ & $3(2)$ & \multirow{5}{*}{0.1270} \\
\hline Normal weight & $104(31)$ & $43(25)$ & $61(37)$ & \\
\hline Overweight & $126(37)$ & $70(40)$ & $56(34)$ & \\
\hline Obese & $103(30)$ & $57(33)$ & $46(28)$ & \\
\hline Missing & 24 & 1 & 23 & \\
\hline \multicolumn{5}{|c|}{ Stage at diagnosis ${ }^{*}, N(\%)$} \\
\hline I & $19(8)$ & $12(10)$ & $7(6)$ & \multirow{5}{*}{0.1253} \\
\hline II & $19(8)$ & $9(7)$ & $10(9)$ & \\
\hline III & $56(24)$ & $35(29)$ & $21(18)$ & \\
\hline IV & $142(60)$ & $65(54)$ & $77(67)$ & \\
\hline Missing & 127 & 53 & 74 & \\
\hline \multicolumn{5}{|c|}{ Cell morphology, $N(\%)$} \\
\hline Leiomyosarcoma & $104(29)$ & $50(29)$ & $54(29)$ & \\
\hline Liposarcoma & $40(11)$ & $20(11)$ & $20(11)$ & \\
\hline Synovial Sarcoma & $12(3)$ & $9(5)$ & $3(2)$ & \\
\hline Other STS ${ }^{* *}$ & $207(57)$ & $95(55)$ & $112(59)$ & \\
\hline \multicolumn{5}{|l|}{ Tumor grade, $N(\%)$} \\
\hline 1 & $24(12)$ & $11(10)$ & $13(13)$ & \multirow{5}{*}{0.8523} \\
\hline 2 & $33(16)$ & $19(18)$ & $14(14)$ & \\
\hline 3 & $142(69)$ & $74(69)$ & $68(69)$ & \\
\hline $4+$ & $8(4)$ & $4(4)$ & $4(4)$ & \\
\hline Missing & 156 & 66 & 90 & \\
\hline \multicolumn{5}{|l|}{ Tumor type, $N(\%)$} \\
\hline Resectable & $105(61)$ & $51(50)$ & $54(61)$ & \multirow{3}{*}{0.8543} \\
\hline Unresectable & $68(39)$ & $34(40)$ & $34(39)$ & \\
\hline Missing & 190 & 89 & 101 & \\
\hline \multicolumn{5}{|c|}{ Baseline ECOG, $N(\%)$} \\
\hline 0 & $59(20)$ & $30(22)$ & $29(18)$ & \multirow{4}{*}{0.0034} \\
\hline 1 & $153(52)$ & $79(49)$ & $74(47)$ & \\
\hline $2+$ & $84(28)$ & $25(19)$ & $59(36)$ & \\
\hline Missing & 67 & 40 & 27 & \\
\hline \multicolumn{5}{|c|}{ ECOG after first-line treatment, $N(\%)$} \\
\hline 0 & $17(15)$ & $17(15)$ & & \\
\hline 1 & $69(59)$ & $69(59)$ & & \\
\hline $2+$ & $30(26)$ & $30(26)$ & & \\
\hline Missing & 58 & 58 & & \\
\hline
\end{tabular}


TABLE 1: Continued.

\begin{tabular}{|c|c|c|c|c|}
\hline Characteristic & Total $(N=363)$ & Treated patients $(N=174)$ & Untreated patients $(N=189)$ & $P$ \\
\hline \multicolumn{5}{|l|}{ Primary site $^{\dagger}$} \\
\hline Head and neck & & $14(8)$ & & \\
\hline Lung & & $9(5)$ & & \\
\hline Liver & & $3(2)$ & & \\
\hline Trunk and viscera & & $25(14)$ & & \\
\hline Retroperitoneal & & $33(19)$ & & \\
\hline Extremity & & $58(33)$ & & \\
\hline Other & & $29(17)$ & & \\
\hline Missing & & $3(2)$ & & \\
\hline
\end{tabular}

SD: standard deviation, BMI: body mass index, and ECOG: Eastern Cooperative Oncology Group Performance Status. P: significance value by Chi-square test. ${ }^{*}$ The stage of disease for each patient is consistent with the descriptions of the AJCC7 classifications of disease.

** Other STS include angiosarcoma of soft tissue, alveolar rhabdomyosarcoma, alveolar soft part sarcoma, fibrosarcoma, Kaposi sarcoma, PNET, pleomorphic rhabdomyosarcoma, clear-cell sarcoma of soft tissue, malignant fibrous histiocytoma, myxofibrosarcoma, malignant phyllodes cystosarcoma, embryonal rhabdomyosarcoma, extraskeletal Ewing tumor, extraskeletal myxoid chondrosarcoma, osteosarcoma, malignant ossifying fibromyxoid tumor, malignant peripheral nerve sheet tumor, hemangiopericytoma, and sarcoma NOS.

${ }^{\dagger}$ Primary sites were captured through chart reviews for the treated patient cohort $(N=174)$ only. Chart reviews were not conducted for the untreated patient $\operatorname{cohort}(N=189)$.

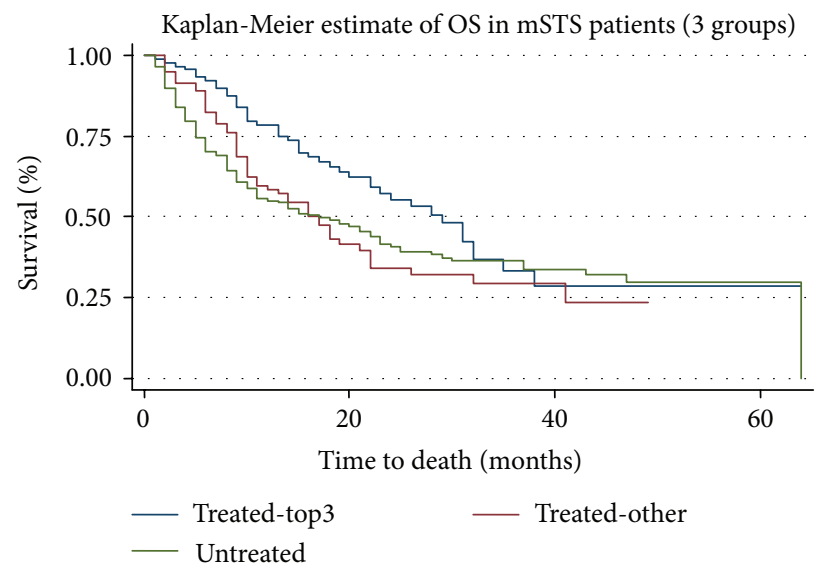

(a)

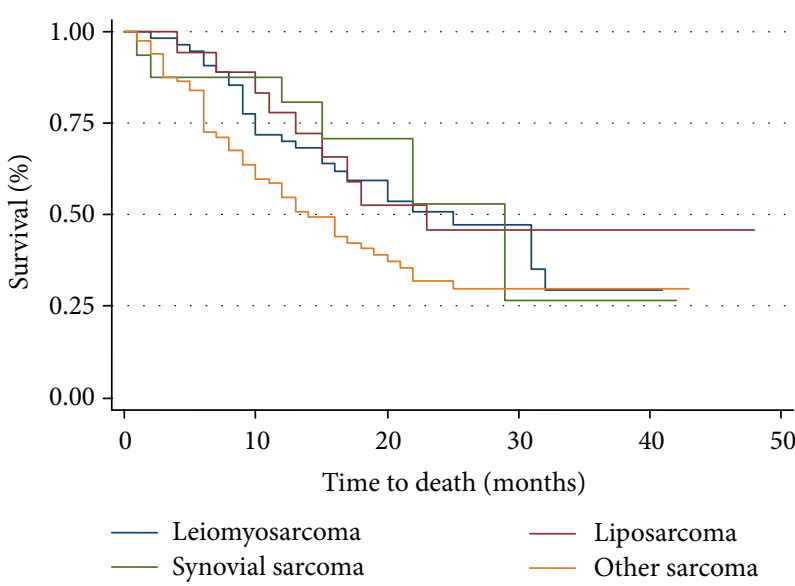

(b)

FIGURE 1: Kaplan-Meier estimates of overall survival in untreated and treated metastatic STS patients. (a) Treated patients were stratified according to histologic subtype. The top three histology subtypes were pooled into one group (treated-top 3), and the remainder were classified as "treated-other." (b) Kaplan-Meier plots for the three most common STS subtypes (leiomyosarcoma, liposarcoma, and synovial sarcoma) in the study population and the remaining subtypes (other sarcoma).

the other STSs (65.5\%) received combination chemotherapy (Supplementary Table 1).

Among the 50 individuals receiving first-line monotherapy, $28 \%$ completed therapy as scheduled, and early discontinuation of therapy was attributable to disease progression in $18 \%$ and drug toxicity in $16 \%$. Among the 129 subjects receiving first-line combination therapy, the corresponding values were $35 \%, 16 \%$, and $13 \%$, respectively. Twelve subjects received second-line monotherapy, and of these 33\% completed therapy, $25 \%$ discontinued therapy early because of disease progression, and $17 \%$ terminated therapy early because of drug toxicity; the corresponding proportions for the 30 patients who received second-line combination chemotherapy were $27 \%, 17 \%$, and $7 \%$, respectively.

3.3. OS and PFS: Unstratified Analyses. Approximately $40 \%$ of the treated and untreated patients died during follow-up.
The median OS of treated patients was 22 (95\% CI, 17 to 29) months whereas for the untreated patients it was 17 (95\% CI, 11 to 23) months (Supplementary Table 2). The percent OS of treated patients at $6,12,24$, and 36 months was $88 \%, 69 \%$, $45 \%$, and $32 \%$, respectively, while that of untreated patients was $70 \%, 55 \%, 41 \%$, and $34 \%$, respectively (Supplementary Table 2).

The OS estimates of patients stratified by STS subtype are shown in Supplementary Table 3 and depicted in Figure 1. Figure 1(a) shows the Kaplan-Meier plots for the OS of two treated subgroups and untreated patients, and Figure 1(b) shows the Kaplan-Meier plots of OS for each of the top three histology subtypes and the other sarcomas. The Kaplan-Meier plots for the top three subtypes were not different from each other (Figure 1(b)) but were different from the other sarcomas and the untreated patients (Figures 1(a) and 1(b)). The median OS for the treated patients with the top three most common 


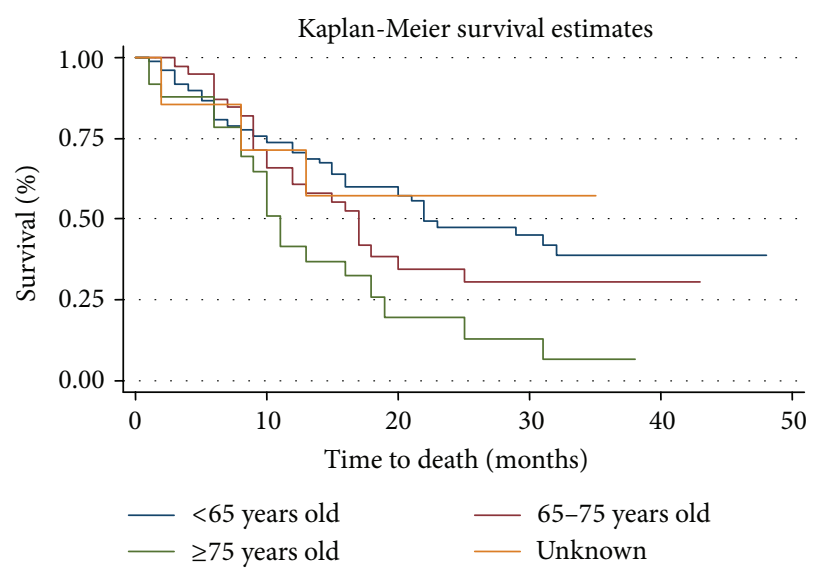

(a)

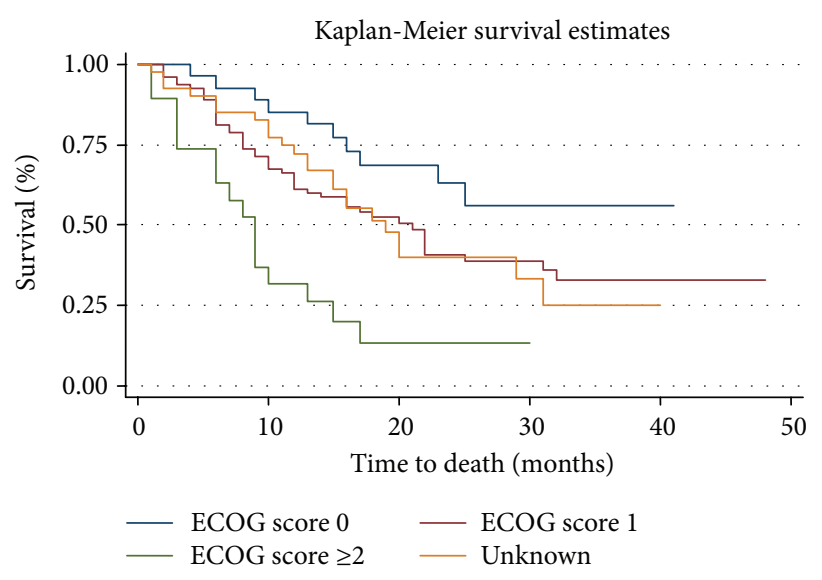

(b)

FIGURE 2: Overall survival by age and ECOG status among mSTS patients starting first-line therapy. (a) Kaplan-Meier plots by age group. (b) Kaplan-Meier plots by baseline ECOG status.

histologic subtypes was 29 (95\% CI, 22 to 35) months. The median for the treated patients with the other histologic subtypes was 17 (95\% CI, 11 to 22) months, similar to that observed in the untreated patients.

The PFS estimates of patients stratified by STS subtype are shown in Supplementary Table 4 and depicted in Supplementary Figure 2. The median overall PFS of treated patients was 11 (95\% CI, 9 to 14) months. The median PFS for treated patients with leiomyosarcoma, liposarcoma, synovial sarcoma, or other sarcomas was 12,18,17, and 7 months, respectively (Supplementary Table 4).

3.4. OS and PFS: Stratified by Line of Therapy. The median OS of patients treated with first-line chemotherapy during the study period was 22 (95\% CI, 17 to 28) months. The mean OS was 24 (95\% CI, 24 to 24 ) months, and the 6-, 12-, 24-, and 36-month OS rates for first-line therapy were 88\% (95\% CI, $82 \%$ to $92 \%$ ), $69 \%$ (95\% CI, $62 \%$ to $76 \%$ ), $45 \%$ (95\% CI, $36 \%$ to $53 \%$ ), and $33 \%$ (95\% CI, $24 \%$ to $42 \%$ ), respectively. The median OS of patients treated with second-line therapy was 11 (95\% CI, 8 to 19) months, whereas the mean OS was 13 ( $95 \%$ CI, 12 to 13) months and the 6- and 12-month OS rates were $70 \%$ (95\% CI, 54 to 82 ) and $44 \%$ (95\% CI, 29 to $58 \%$ ), respectively.

The median PFS of patients treated with first-line chemotherapy during the study period was 11 (95\% CI, 9 to 14) months. The mean PFS was 15 (95\% CI, 14 to 15) months, and the 6-, 12-, 24- and 36-month PFS rates for first-line therapy were $66 \%$ (95\% CI, $58 \%$ to $72 \%$ ), $46 \%$ (95\% CI, $38 \%$ to $54 \%$ ), $28 \%$ (95\% CI, $20 \%$ to $36 \%$ ), and $17 \%$ (95\% CI, $10 \%$ to $26 \%$ ), respectively (Supplementary Table 4 ). The median PFS of patients treated with second-line therapy was 9 (95\% CI, 7 to 12) months, whereas the mean PFS was 11 (95\% CI, 10 to 11) months, and the 6-and 12-month PFS rates were 64\% (95\% CI, $47 \%$ to $77 \%$ ) and $35 \%$ ( $95 \%$ CI, $20 \%$ to $50 \%$ ), respectively.

3.5. Overall Survival by Age. Among those receiving first-line therapy, $102(58.9 \%), 39(22.5 \%)$, and $25(14.5 \%)$ were $<65$, $65-75$, and $\geq 75$ years old, respectively (age was unknown in 7 subjects (4.1\%)), and the corresponding values for those receiving second-line therapy were $26(61.9 \%), 9(21.4 \%)$, and $3(7.1 \%)$ (age was unknown in 4 (9.5\%) subjects). Among those receiving first-line monotherapy $(n=50), 40 \%, 20 \%$, and $36 \%$ were $<65,65-75$, and $\geq 75$ years old, respectively, and corresponding values for those receiving first-line combination therapy $(n=129)$ were $65.9 \%, 22.5 \%$, and $7.8 \%$, respectively. There was a similar age distribution by secondline monotherapy and combination chemotherapy.

The Kaplan-Meier analyses for OS by age at the start of first-line therapy are shown in Figure 2(a). The mean OS for those initiating first-line therapy at ages $<65,65$ to 75 , and $>75$ years was 21 (95\% CI, 21 to 21$), 16$ (95\% CI, 16 to 16 ), and 13 (95\% CI, 13 to 14) months, respectively. The mean PFS for those initiating first-line therapy at ages $<65,65-75$, and $>75$ years was 15 (95\% CI, 15 to 15$), 14$ (95\% CI, 14 to 15 ), and 12 (95\% CI, 11 to 13) months, respectively (data not shown).

3.6. Overall Survival by ECOG Status. Among those receiving first-line therapy, 29 (16.7\%), 83 (47.7\%), and 19 (10.9\%) had an ECOG status of 0, 1, and 2, respectively (ECOG status was unknown in 42 subjects (24.1\%)). The Kaplan-Meier analyses of OS by baseline ECOG status are shown in Figure 2(b). The mean OS for those initiating first-line therapy by ECOG status of 0,1 , and $\geq 2$ was 21 ( $95 \%$ CI, 20 to 21 ), 20 (95\% CI, 20 to 20 ), and 9 ( $95 \%$ CI, 9 to 10) months, respectively. The corresponding mean PFS values were 17 (95\% CI, 16 to 18 ), 15 (95\% CI, 15 to 15 ), and 8 ( $95 \%$ CI, 7 to 8 ) months, respectively.

Among those receiving first-line monotherapy $(n=$ 50 ), $6 \%, 50 \%$, and $20 \%$ had ECOG scores of 0,1 , and $\geq 2$, respectively, and the corresponding values for those receiving first-line combination therapy were $20.9 \%, 46.5 \%$, and $6.9 \%$, respectively. The relative distribution of ECOG scores by second-line monotherapy and combination chemotherapy was similar.

3.7. Overall Survival by Metastasis Site. Among subjects who initiated first-line therapy, the most common sites for metastasis were lung (36.9\%), trunk and viscera (10.4\%), 
retroperitoneal (8.6\%), and multiple metastatic sites (8.6\%). The mean OS in these patients was 15 (95\% CI, 14 to 15$), 16$ (95\% CI, 15 to 16), 17 (95\% CI, 16 to 17), and 20 (95\% CI, 20 to 21$)$ months, respectively. The mean PFS was 10 (95\% CI, 10 to 10$), 14$ (95\% CI, 14 to 15$), 14$ (95\% CI, 14 to 15$)$, and 17 (95\% CI, 17 to 18 ) months, respectively (data not shown).

\section{Discussion}

This retrospective observational study conducted in a large community oncology network yielded six key findings. First, STS histologic subtypes were similar between the treated and untreated subjects (i.e., tumor subtypes, stage, and grade). Leiomyosarcoma, liposarcoma, and synovial sarcoma were the top three most common histologic subtypes in this patient population, consistent with the previously reported distribution patterns $[7,15,16]$. Second, while tumor characteristics were similar, treated patients differed from untreated individuals in three respects: they were younger, a greater proportion was men, and they had lower ECOG scores.

Third, our study revealed insights into chemotherapy treatment patterns for mSTS in the community setting. Among patients receiving first-line chemotherapy, 75\% and $\sim 25 \%$ received combination and monotherapy, respectively. Among patients with leiomyosarcoma, the most frequent form of mSTS in our study population, $84 \%$ received combination therapy. The most common combination regimens in the overall study population were doxorubicin plus ifosfamide (29\%) and docetaxel plus gemcitabine (24\%). This choice is expected based on prevailing clinical practice [18]. For example, gemcitabine with docetaxel has been found to be active in leiomyosarcoma of uterine and gastrointestinal origin: a phase 2 study reported a higher response rate in this subtype for combination docetaxel plus gemcitabine versus gemcitabine alone (32\% versus $27 \%$, resp.) and significantly improved progression-free survival (6.3 versus 3 months, resp.) [19]. Although the benefits of chemotherapy for mSTS and the use of combination versus single agent chemotherapy for mSTS are unclear, combination chemotherapy is generally an accepted practice standard in the USA [2, 3]. Further research is needed to determine the optimal dosing and tolerability of these regimens in these patients. Even though the primary focus of this study was the chemotherapy treatment patterns in the community setting, future research should also explore the effects of surgery and radiation on these patients.

Fourth, the median OS of treated and untreated patients prior to accounting for tumor subtype was 22 and 17 months, respectively. A retrospective analysis of seven clinical trials of chemotherapy-naïve patients with advanced STS revealed that the overall median survival time of the 2,185 patients in the therapy arms was 51 weeks [16]; similar data were observed in more recent clinical trials $[15,17]$. The basis for the longer overall survival times in our study subjects treated in the community compared with results from clinical trials is unclear and needs further investigation. However, consistent with previous studies $[15,16]$, patients with the top three histologic subtypes had better outcomes than those with the other subtypes. Among patients with the top three histologic subtypes, the median overall survival was 29 months, with similar trends observed for PFS. This observation could relate to differences in the underlying biology of these three tumors, such that, compared to the heterogeneous group of STS that were pooled into one group (other), the top three histologic types may be more responsive to therapy and/or have less aggressive disease characteristics. Another possibility could be that a slightly greater proportion of patients with the top three subtypes were treated with combination therapy (78.3\%) compared to those with the other STS subtypes (65.5\%). Selection biases could have contributed to these differences in outcomes by histology subtype. However, this was less likely, as we found that ages and ECOG scores between treated patients were similar by histology subtype.

Fifth, consistent with prior studies [16], we also found that younger age and lower ECOG scores were associated with longer OS in treated patients. While there was a significant difference in mean age and baseline ECOG scores between treated and untreated patients, larger cohorts in future studies will be needed to properly control for the influence these factors may have on OS and PFS outcomes. Finally, lung was the most common site for metastasis, and those with lung metastasis had shorter OS and PFS compared to subjects with metastasis to other sites.

Due to the retrospective, observational design of this study, there are some limitations worth noting. While the use of a large geographically dispersed cohort of communitybased patients provides confidence that our results may potentially be able to be generalized, patterns of care within the MSH/USON network may differ to some extent from community-based treatment patterns in general. This may be due to the encouragement given to oncologists by the MSH/ USON network administration to base their therapy decisions on evidence-based treatment guidelines. Additional limitations of this study include the exclusion of patients from specific sites in the MSH/USON network from the study sample because only partial iKnowMed EHR capabilities were adopted at these sites; it is possible that patterns of care at these specific sites may differ to some extent from the remainder of the sites. Another limitation includes the lack of differentiation of STS subtypes. Our EHR and chart review did not capture the different variants of liposarcoma or other important subtypes. Selection bias of subtype variants may have influenced response and survival rates and should be considered in future research. Also, escalation in LOT was used as a proxy for disease progression. Since there may be some delay from disease progression to when patients received their next line of chemotherapy, the progression-free survival may be overestimated. In addition, the iKnowMed data are collected for clinical practice reasons and not for research purposes. This may limit the standardization of the data collection methods and instruments as well as the reporting practices of the physician. Finally, our study was not designed to compare the efficacy of monotherapy versus combination therapy or determine the factors that associate with poorer clinical outcomes in STS. Thus, the inferences of this observational study need confirmation in randomized, controlled trials of adequate size prospectively designed to address these questions. 


\section{Conclusions}

To our knowledge, this is the first study to use a cancerspecific database to capture "realworld" clinical data on patients with mSTS in the community-based setting. The results of this study are strengthened by the large sample size and potentially greater diversity of care compared with clinical trial or tertiary care academic settings. Taken together, by examining a community-based, cancer-specific EHR, we provide new insights into the epidemiology, treatment patterns, and outcomes of patients with mSTS who received care outside of an academic or clinical trial setting in the USA and elaborate on their implications for future clinical research.

\section{Conflict of Interests}

Employment or Leadership Position: E. Nadler (The US Oncology Network). Employment or Leadership Position: R. Borker and M. Hackshaw (GlaxoSmithKline). Consultant or Advisory Role: E. Nadler (The US Oncology Network). Stock Ownership: R. Borker and M. Hackshaw (GlaxoSmithKline). Honoraria: None. James Ewing, MD, and Eric Nadler, MD, are Medical Directors of Health Informatics at US Oncology Research, McKesson Specialty Health, Department of Oncology, Baylor Charles A. Sammons Cancer Center and Baylor University Medical Center at Dallas. Rohit Borker and Michelle D. Hackshaw are employees of GlaxoSmithKline.

\section{Acknowledgments}

The research was supported by GlaxoSmithKline. The authors would like to thank Debra Rembert and Mark Yap for chart data extraction support and Brooke Middlebrook for editorial support.

\section{References}

[1] E. C. Borden, L. H. Baker, R. S. Bell et al., "Soft tissue sarcomas of adults: state of the translational science," Clinical Cancer Research, vol. 9, no. 6, pp. 1941-1956, 2003.

[2] G. D. Demetri, L. H. Baker, R. S. Benjamin et al., "Soft tissue sarcoma," Journal of the National Comprehensive Cancer Network, vol. 5, pp. 364-399, 2007.

[3] P. G. Casali, L. Jost, S. Sleijfer, J. Verweij, and J.-Y. Blay, "Soft tissue sarcomas: ESMO clinical recommendations for diagnosis, treatment and follow-up," Annals of Oncology, vol. 19, no. 2, pp. ii89-ii93, 2008.

[4] A. Jemal, R. Siegel, J. Xu, and E. Ward, "Cancer statistics, 2010," CA: A Cancer Journal for Clinicians, vol. 60, no. 5, pp. 277-300, 2010.

[5] N. H. Segal, P. Pavlidis, C. R. Antonescu et al., "Classification and subtype prediction of adult soft tissue sarcoma by functional genomics," The American Journal of Pathology, vol. 163, no. 2, pp. 691-700, 2003.

[6] M. A. Clark, C. Fisher, I. Judson, and J. Meirion Thomas, "Softtissue sarcomas in adults," The New England Journal of Medicine, vol. 353, no. 7, pp. 701-711, 2005.

[7] J. N. Cormier and R. E. Pollock, "Soft tissue sarcomas," Ca: A Cancer Journal for Clinicians, vol. 54, no. 2, pp. 94-109, 2004.
[8] S. A. Lietman, "Soft-tissue sarcomas: overview of management, with a focus on surgical treatment considerations," Cleveland Clinic Journal of Medicine, vol. 77, supplement 1, pp. S13-S17, 2010.

[9] T. F. DeLaney, L. Kepka, S. I. Goldberg et al., "Radiation therapy for control of soft-tissue sarcomas resected with positive margins," International Journal of Radiation Oncology Biology Physics, vol. 67, no. 5, pp. 1460-1469, 2007.

[10] R. Wesolowski and G. T. Budd, "Use of chemotherapy for patients with bone and soft-tissue sarcomas," Cleveland Clinic Journal of Medicine, vol. 77, supplement 1, pp. S23-S26, 2010.

[11] V. H. Bramwell, D. Anderson, and M. L. Charette, "Doxorubicin-based chemotherapy for the palliative treatment of adult patients with locally advanced or metastatic soft tissue sarcoma," Cochrane Database of Systematic Reviews, no. 3, Article ID CD003293, 2003.

[12] K. Antman, J. Crowley, S. P. Balcerzak et al., "An intergroup phase III randomized study of doxorubicin and dacarbazine with or without ifosfamide and mesna in advanced soft tissue and bone sarcomas," Journal of Clinical Oncology, vol. 11, no. 7, pp. 1276-1285, 1993.

[13] A. le Cesne, I. Judson, D. Crowther et al., "Randomized phase III study comparing conventional-dose doxorubicin plus ifosfamide versus high-dose doxorubicin plus infosfamide plus recombinant human granulocyte-macrophage colony- stimulating factor in advanced soft tissue sarcomas: a trial of the european organization for research and treatment of cancer/soft tissue and bone sarcoma group," Journal of Clinical Oncology, vol. 18, no. 14, pp. 2676-2684, 2000.

[14] S. Jelić, V. Kovčin, N. Milanović et al., "Randomised study of high-dose epirubicin versus high-dose epirubicin-cisplatin chemotherapy for advanced soft tissue sarcoma," European Journal of Cancer Part A, vol. 33, no. 2, pp. 220-225, 1997.

[15] B. L. Samuels, S. Chawla, S. Patel et al., "Clinical outcomes and safety with trabectedin therapy in patients with advanced soft tissue sarcomas following failure of prior chemotherapy: results of a worldwide expanded access program study," Annals of Oncology, vol. 24, pp. 1703-1709, 2013.

[16] M. van Glabbeke, A. T. van Oosterom, J. W. Oosterhuis et al., "Prognostic factors for the outcome of chemotherapy in advanced soft tissue sarcoma: an analysis of 2,185 patients treated with anthracycline- containing first-line regimens-a European organization for research and treatment of cancer soft tissue and bone sarcoma group study," Journal of Clinical Oncology, vol. 17, no. 1, pp. 150-157, 1999.

[17] W. T. van der Graaf, J. Y. Blay, S. P. Chawla et al., "Pazopanib for metastatic soft-tissue sarcoma (PALETTE): a randomised, double-blind, placebo-controlled phase 3 trial," The Lancet, vol. 379, pp. 1879-1886, 2012.

[18] R. G. Maki, "Gemcitabine and docetaxel in metastatic sarcoma: past, present, and future," Oncologist, vol. 12, no. 8, pp. 9991006, 2007.

[19] R. G. Maki, J. K. Wathen, S. R. Patel et al., "Randomized phase II study of gemcitabine and docetaxel compared with gemcitabine alone in patients with metastatic soft tissue sarcomas: results of sarcoma alliance for research through collaboration study 002," Journal of Clinical Oncology, vol. 25, no. 19, pp. 2755-2763, 2007. 


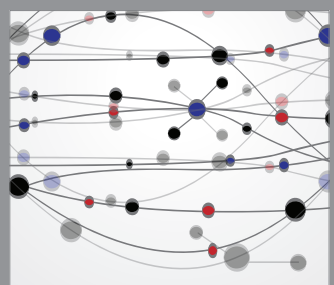

The Scientific World Journal
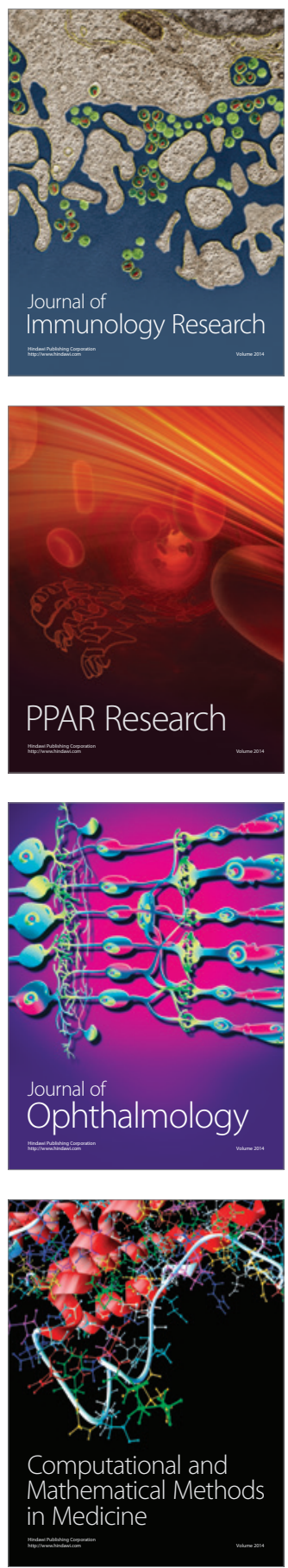

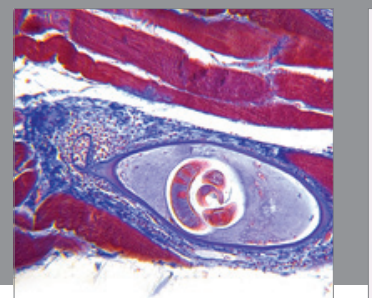

Gastroenterology

Research and Practice
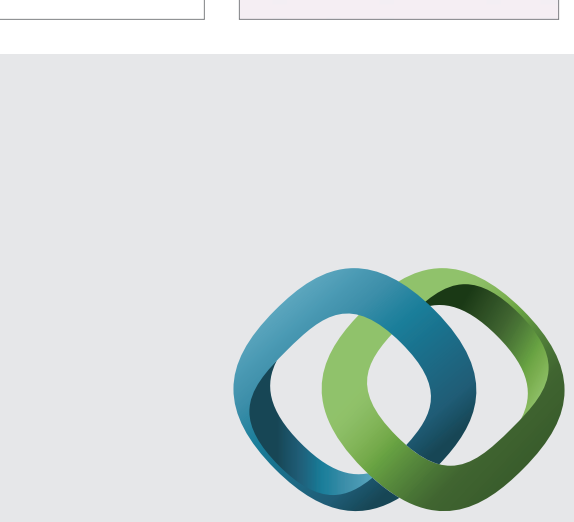

\section{Hindawi}

Submit your manuscripts at

http://www.hindawi.com
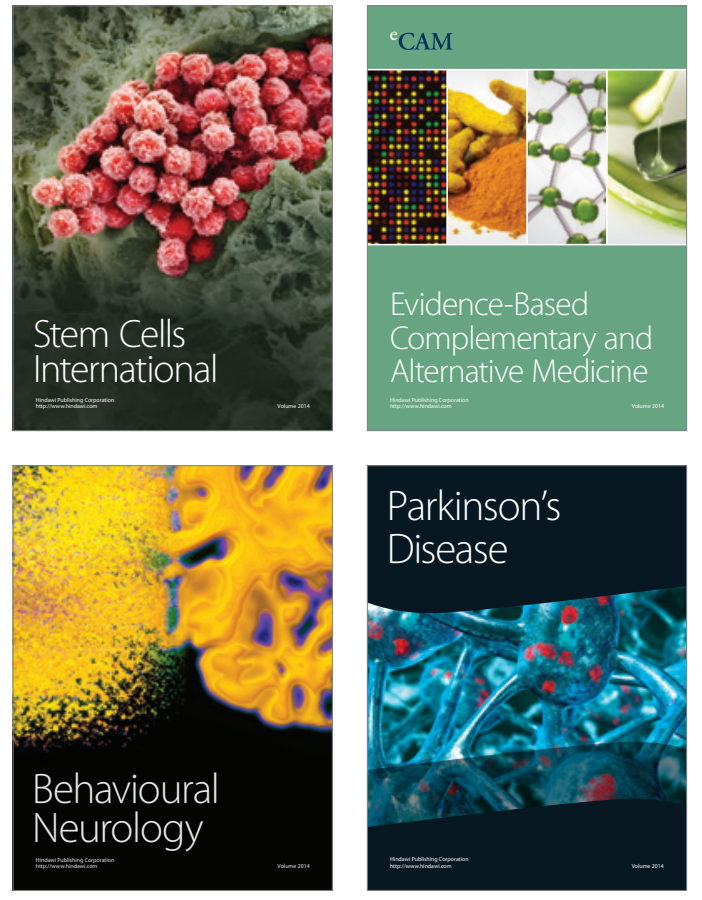
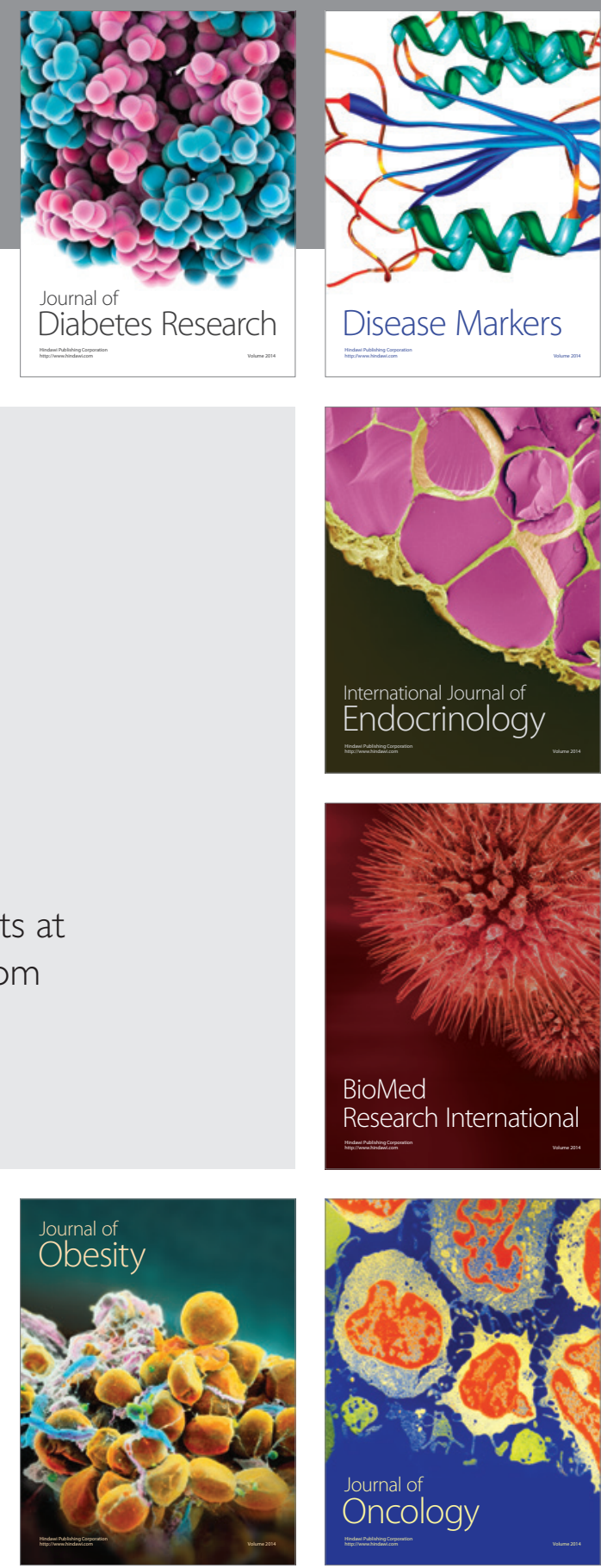

Disease Markers
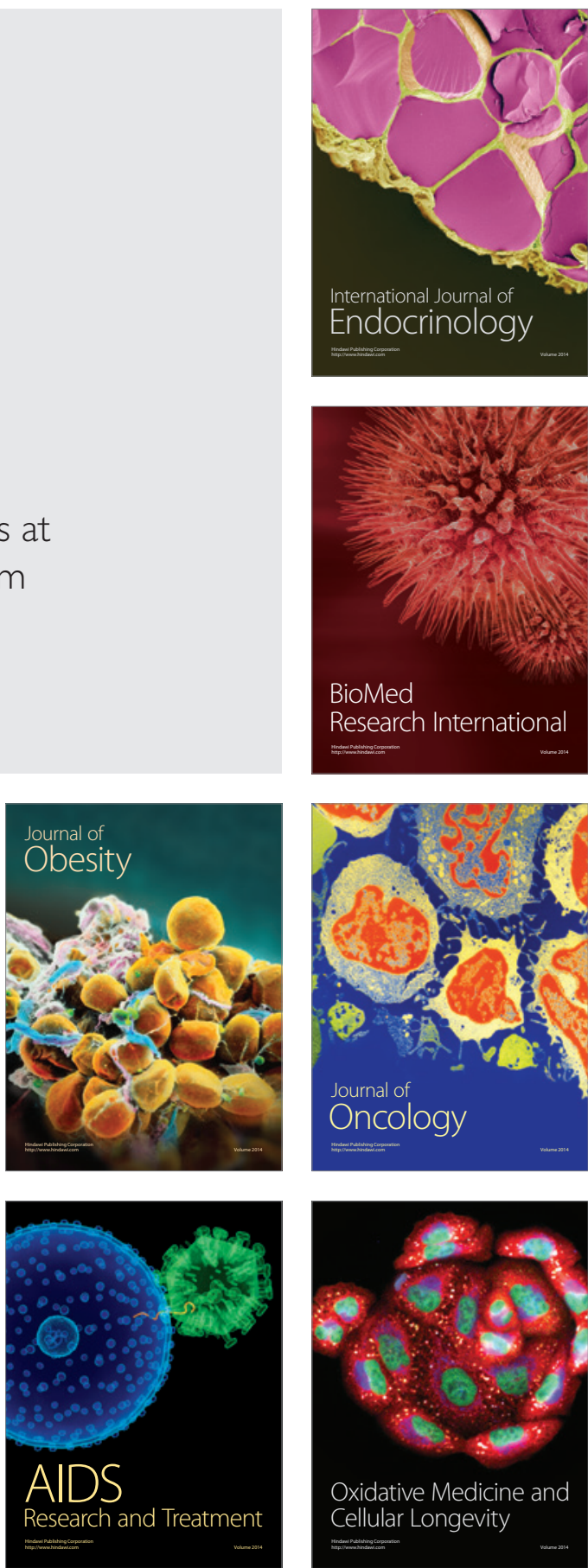